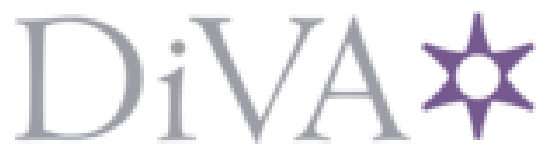

http://www.diva-portal.org

This is the published version of a paper presented at The 29th IEEE International Conference on Robot \& Human Interactive Communication (RO-MAN 2O2O).

Citation for the original published paper:

Torre, I., Le Maguer, S. (2020)

Should robots have accents?

In:

N.B. When citing this work, cite the original published paper.

Permanent link to this version:

http://urn.kb.se/resolve?urn=urn:nbn:se:kth:diva-283665 


\title{
Should robots have accents?
}

\author{
Ilaria Torre ${ }^{1}$ and Sébastien Le Maguer ${ }^{2}$
}

\begin{abstract}
Accents are vocal features that immediately tell a listener whether a speaker comes from their same place, i.e. whether they share a social group. This in-groupness is important, as people tend to prefer interacting with others who belong to their same groups. Accents also evoke attitudinal responses based on their supposed prestigious status. These accent-based perceptions might affect interactions between humans and robots. Yet, very few studies so far have investigated the effect of accented robot speakers on users' perceptions and behaviour, and none have collected users' explicit preferences on robot accents. In this paper we present results from a survey of over 500 British speakers, who indicated what accent they would like a robot to have. The biggest proportion of participants wanted a robot to have a Standard Southern British English (SSBE) accent, followed by an Irish accent. Crucially, very few people wanted a robot with their same accent, or with a machine-like voice. These explicit preferences might not turn out to predict more successful interactions, also because of the unrealistic expectations that such human-like vocal features might generate in a user. Nonetheless, it seems that people have an idea of how their artificial companions should sound like, and this preference should be considered when designing them.
\end{abstract}

\section{INTRODUCTION}

Designing the voices of artificial agents, including robots, is no easy task. Apart from being functional (intelligible, relevant, etc.), they should also be appealing in a way that the user will continue to use the services that they provide. This is a difficult problem, since individual differences will likely come into play. For example, some people might prefer their artificial companion to have a female voice, others a male voice, others the voice of a celebrity, etc. Currently, the market of digital personal assistants is monopolised by interfaces that as default have a synthetic, female, US-accented voice [1, 2]. Apart from raising concerns of the younger generations forming associations of servitude between these assistants and female voices (as recently pointed out by UNESCO [3]), this monopoly might also not appeal to every user. How do these kinds of voices resonate, for example, with users who are not from the USA? Sociolinguistic research states that people show in-group favouritism, i.e. they prefer interacting with people from their same social group [4]. Accent, the way a person pronounces words in a certain language [5], is one way in which people can tell whether someone is coming from their same place, and

\footnotetext{
*This work was supported by CogNovo FP7-PEOPLE-2013-ITN- 604764 (https://CogNovo.eu), a project funded by Marie Skłodowska- Curie Actions. The first author is funded by a WASP Expedition Project on Correctby-design and Socially Acceptable Autonomy (CorSA)

${ }^{1}$ Ilaria Torre is with KTH Royal Institute of Technology, Stockholm, Sweden ilariat@kth. se

${ }^{2}$ Sébastien Le Maguer is with the ADAPT Centre and Trinity College Dublin, Dublin, Ireland lemaguesetcd.ie
}

thus share one social group with them. Accented voices also evoke implicit attitudinal responses based on national or regional stereotypes, which can be positive or negative [6]. Therefore, the accent of an artificial agent is a parameter that could be modified to evoke the desired attitudes and feelings in its users. Yet this is rarely exploited in machine design [7]. Besides, accented voices might inspire a certain realism in the mind of the users. This might not be a problem in the case of disembodied user interfaces, such as digital personal assistant, but it could create a mismatch in the realism between the voice and visual appearance of embodied agents, such as robots.

Robots have the potential of establishing more rapport with a user than bodyless agents, due to their social presence [8, 9]. This is important in cases where the robot needs to fulfil a companionship function, for example in the case of assistive robots for the elderly. Having a specific accent might make these robots warmer and more acceptable for these vulnerable users. Nonetheless, this problem is relatively new, and it is necessary to start from the beginning. So, in this paper, we ask: what is the users' view on giving accents to robots?

\section{BACKGROUND}

Accents can be defined as any systematic differences in pronouncing the sounds of a language, that people belonging to a certain group (geographic or social) have in common [5]. Thus, every speaker has an accent. However, in countries such as the United Kingdom, where there is a defined 'standard accent' (Standard Southern British English, SSBE), it is common to hear people say that they "don't have an accent". This is considered as belonging to a higher social class than people who speak with a distinct regional accent [10]. Indeed, long-term Sociolinguistic studies on accent perception have shown that standard accents are generally considered prestigious and trustworthy, while regional and non-native accents are perceived more negatively [11, 12].

Hearing someone's accent is unavoidable: when we hear someone speak, we can immediately tell whether they speak like us or not. Unconsciously, this places the speaker either inside our social group, or outside of it. This in-group vs. out-group localisation then brings forward a series of attitudinal responses towards that speaker [13]. For example, a preference to listening to one's native language emerges from the first few months of life [14]. Then, children start to trust native-accented speakers around 4-5 years of age [4]. Later on, this implicit preference shifts to an in-group or familiarity preference for the accent of one's social group [15]. 
This apparent contradiction between higher attitudinal evaluations for standard accents and preference for one's own accent seems to resolve in terms of which traits are being evaluated. For example, speakers of South Welsh and Somerset varieties of English showed an in-group preference effect for 'informal' speaker traits, such as good looks and humour, while at the same time showing a preference for the prestigious RP accent for 'formal' traits, such as competence [6].

Thus, on the one hand, accents contribute to create social groups. Accent-based social categorisations and feelings of in-groupness are believed to be even stronger than those due to gender or ethnicity [16]. An evolutionary explanation for these findings is that our ancestors likely had to defend themselves more often from neighbouring social groups, which might have looked very similar, but still sounded different, as accents develop in small geographic areas [16]. On the other hand, many languages have a prestigious, standard accent, which generally elicits positive first impressions, especially related to trust and competence [6, 11]. In turn, these first impressions shape future behaviour towards the accented speaker [12].

While human speakers often cannot choose which accent to speak with (nor should they have to!), as designers of speaking machines we can choose which accents to give to our artificial companions. One option could be to give them the same accent as their user, to increase feelings of in-groupness [17]. In-groupness need not be innate; previous studies have shown that it is possible to prime it [18], even with artificial agents [19]. Generating such a connection with robots might be beneficial for Human-Robot teams, where humans and machines have to collaborate at potentially high stakes [20]. It might also be particularly beneficial in those situations where humans and machines need to establish rapport, for example where the robot is a companion.

Another option could be to give artificial agents a prestigious accent, in order to evoke feelings of trust, knowledge, and reliability [6]. This might be particularly important in cases where users need to follow robot recommendations, for example when the robot is supervising a medical treatment. Having the robot speak with a prestigious accent might increase human trust and compliance.

However, accented robots have received little attention so far. Within the field of Human-Robot Interaction (HRI), researchers are not always aware of the importance of giving a multi-faceted voice to their robots, including features such as accent. In a recent survey, [21] asked researchers who had run studies with speaking robots why they had chosen a particular robot voice. The majority of the 18 respondents stated that they had chosen one because of its convenience and availability; six researchers explained that they had adjusted some features of the voice, such as pitch and speech rate, to suit their needs; only one researcher mentioned that they had looked for a specific accent.

Previous studies using disembodied artificial agents found that participants engaged more in interaction with agents who spoke in the same accent as themselves, and rated them as warmer and more trustworthy [22]. Even without voices, giving robots a regional identity affected their perceived warmth and competence: [23] ran an experiment with four robots, which were given four different 'nationalities'. These nationalities were chosen because of their stereotypical low or high warmth and competence. They found that the stereotypes transferred to the robots. In another study, participants were told that a robot was either built in New York, or in Hong Kong; subsequently, participants judged the former to be more knowledgeable about New York, and the latter to be more knowledgeable about Hong Kong [24]. Thus, giving robots regional identities might make the interaction more effective.

Research from [25] on the Arabic language also highlights the interaction between accent and perceived capability in Human-Robot Interaction: participants believed that robots with the same regional accent as theirs were more credible - when the robots were knowledgeable - than those with a standard accent, whereas robots with standard accents were perceived to be more credible when the robots had little knowledge. Similar interactions are likely with other languages, too. [17] ran two experiments comparing synthesised British, American, and New Zealand English accents. In the first experiment, participants from New Zealand explicitly rated the disembodied UK accent more positively than the US one, while their own New Zealand accent was not rated significantly differently to either of the other two accents. In the second experiment, participants were told by a healthcare robot, speaking with one of the three accents, how to take blood pressure measurements on themselves. Participants reported feeling more positive emotions towards the New Zealand-accented robot than the US-accented robot, and thought that the New Zealand robot performed better. Thus, while in this case participants might have had explicit preferences for the prestigious accent (UK English), when it came to actually interacting with the robot, in-group favouritism emerged (New Zealand English).

However, giving human accents to robots also raises the issue of voice naturalness. Interacting with a specific accented-robot might make users form first impressions of human-likeness and human-like abilities. These however might not be reflective of the actual capabilities of the system, and this mismatch risks endangering future interactions [26, 27, 24, 28]. Also, a robot with a natural-sounding voice but machine-like appearance might seem contradictory and elicit feelings of eeriness [29].

From this brief review of the literature on accents and speaking robots, we can identify a few arguments on whether to give robots accents or not, and, if so, which accents:

- Social and evolutionary theories suggest that giving a robot the same accent as its user might create a feeling that robot and user belong to the same social group, and thus the human-robot interpersonal relationship might be enhanced;

- Studies from Sociolinguistics suggest that giving a robot a prestigious accent might increase trust and compliance in the robot; 


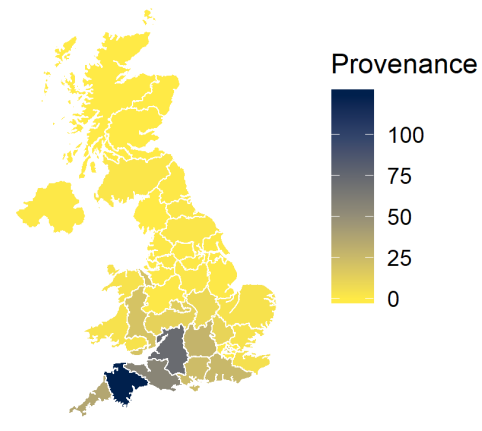

Fig. 1. Map of the UK highlighting the region of origin of the survey participants.

- Practical considerations from the field of HRI suggest that equipping robots with human-like features, such as human accents, might create unrealistic expectations in a user, who might then react more negatively when these expectations are not met.

Given the limited amount of data on humans interacting with accented robots, we believe that a first step into determining whether to give robots specific accents or not is to ask their potential users. This is the aim of this paper: to present the results of a survey conducted with hundreds of participants in the UK, who stated their preference for a robot accent.

\section{METHOD}

\section{A. Participants}

The survey respondents were 503 British people who participated in a series of unrelated experiments in a UK university over a 3 -year period. Of these, 375 identified as female, and 128 as male. They were aged 18-69, with the vast majority $(83 \%, \mathrm{~N}=415)$ being in the $18-22$ age range. All of the respondents were native British English speakers, with the following self-reported regional identities: southwest England (58\%), southeast England (22\%), Midlands (8\%), Wales (5\%), East of England (3\%), with participants from northeast England, northwest England, Scotland, Northern Ireland, and the Channel Islands comprising all of the remaining $4 \%$. The regional provenance of the participants is illustrated in Fig. 1

\section{B. Procedure}

Participants simply answered an open-ended question: "What accent would you like a robot to have?". There were no character limits for the answer. The question was presented on a computer screen. After answering the question, participants filled in a short demographics questionnaire about their gender, age, city of origin, accent, and accent they were most exposed to while growing up. To avoid confusion, the city of origin was described as the place where participants grew up. The total duration of the survey was approximately 5 minutes.

\section{Data Cleaning}

The question was open-ended, so we re-coded the answers to fit in broad categories (e.g. 'West Country' and 'South West' would both be coded as 'South West'; labels such as 'English', 'British', 'RP' would be coded as 'SSBE', etc.). Fig. 2 shows these standardised answers from all the participants who answered this question. Furthermore, we computed an additional variable, the distance (in $\mathrm{km}$ ) from participants' home town to the place corresponding to the accent that they wanted the robot to have. This variable is null in cases where the desired accent is not described geographically (e.g. people who wanted a 'soft' accent). In cases where the desired accent is not a very specific location (e.g. 'Australia' rather than 'Brisbane'), the geometric centre of the indicated location was taken to calculate the distance in $\mathrm{km}$. As can be seen from Fig. 2. many people indicated that they wanted the robot to have a Standard Southern British English accent. This denomination is by definition not linked to any geographic location in the UK, being rather a social accent [6]. However, it is thought to have originated in the South East of England, which is a region that comprises the counties of Berkshire, Buckinghamshire, East Sussex, Hampshire, the Isle of Wight, Kent, Oxfordshire, Surrey and West Sussex. Therefore, we ascribed the geometric centre of the South-East of England, roughly corresponding to the town of Guildford, Surrey, to all the desired accents described as 'SSBE/RP/English/British/Standard English' and similar. Finally many participants stated that they did not know what their own accent was, or that they had 'no accent'. As previously mentioned, many people in the UK and in other countries with standard accents commonly refer to themselves as not having an accent. Of course, from a linguistic point of view, this is not possible [5]. Due to this common interpretation, for the purposes of coding our data, we considered participants' place of origin as a proxy for their accent, or in any case for an accent that they would be very familiar with.

\section{RESUlTS}

Of the 503 participants, 4 did not answer the question. Of the remaining 499, $182(36.5 \%)$ answered that they would like a robot to have an SSBE accent. The second most frequent answer was Irish accent $(\mathrm{N}=56,11.2 \%)$, followed by Australian $(\mathrm{N}=30,6 \%)$, London $(\mathrm{N}=29$, $5.8 \%)$, South West England $(\mathrm{N}=23,4.6 \%)$, 'neutral' $(\mathrm{N}$ $=22,4.4 \%)$, Welsh $(\mathrm{N}=15,3 \%)$, American $(\mathrm{N}=13$, $2.6 \%)$, 'any accent' $(\mathrm{N}=12,2.4 \%)$, Scottish $(\mathrm{N}=12$, $2.4 \%)$, Northern British $(\mathrm{N}=11,2.2 \%)$, 'machine-like' $(\mathrm{N}$ $=10,2 \%)$, Italian $(\mathrm{N}=9,1.8 \%)$, French $(\mathrm{N}=6,1.2 \%)$, Newcastle $(\mathrm{N}=6,1.2 \%)$, Spanish $(\mathrm{N}=5,1 \%)$, 'the same accent as my own' $(\mathrm{N}=4,0.8 \%)$, Liverpool $(\mathrm{N}=4,0.8 \%)$. Some people did not answer with the name of an accent, but described the way they would like a robot to speak. Most of these were about the voice being calm and clear 
$(\mathrm{N}=9,1.8 \%)$; the others, e.g. 'happy', 'fast', 'friendly' were labelled as 'other voice quality' $(\mathrm{N}=6,1.2 \%)$. The remaining participants answered with very different accents (Yorkshire, Oxford, Northern Irish, Midlands, Lancashire, Birmingham, Cheshire, Cockney, Essex, Texan, Latin American, Indian, Canadian, Slovakian, Jamaican, South African, Finnish, Georgian) which were labelled, respectively, as 'other UK accent' $(\mathrm{N}=19,3.8 \%)$ and 'other non-UK accent' $(\mathrm{N}=16,3.2 \%)$. These answers are shown in Fig. 2. Although the majority of participants were from South West and South East England, we also grouped accent preferences based on participants' region of origin, to see if, for example, people from the Northern regions had different accent preferences than people from the Southern regions. However, the trends seem to not change on a region-by-region basis. Plots for each region can be accessed here

In terms of distance from the participants' city of origin, we excluded data from 3 participants, who did not specify what city they were from. Also, we excluded data from the participants who did not describe the robot accent in terms of a geographic location (e.g. 'machine-like', 'any accent' or 'friendly'). Thus, for this analysis we only retained data from 457 participants. Their answers varied widely, from people reporting that they wanted a robot to have the accent of their same city (corresponding to a distance of $0 \mathrm{~km}$ ) to people wanting an accent from Canada or Australia (thousands of $\mathrm{km}$ away). Therefore, we grouped these distances in ranges, as shown in Fig. 3. As can be seen from the Figure, the majority of participants wanted an accent originating from a place different than their place of origin. Specifically, 76 participants $(16.6 \%)$ indicated a location less than $100 \mathrm{~km}$ away from their home town, $95(20.8 \%)$ a location between 100 and $200 \mathrm{~km}$ away, $79(17.3 \%)$ a location between 200 and $300 \mathrm{~km}$ away, $53(11.6 \%)$ a location between 300 and $400 \mathrm{~km}$ away, $27(5.9 \%)$ a location between 400 and 500 $\mathrm{km}$ away, and $103(22.5 \%)$ a location over $500 \mathrm{~km}$ away. Only 24 participants $(5.2 \%)$ said that they wanted the robot to have the accent of their same place of origin, i.e. $0 \mathrm{~km}$ away.

Finally, we looked at whether people indicated a bigger or smaller distance based on their demographic characteristics (gender and age). We ran a linear regression model with distance from home town as dependent variable, and gender and age as predictors. There was no main effect for gender or age, and no interaction $(F(3,453)=1.16$, all $p>.05)$.

\section{DISCUSSION}

We conducted a survey asking British participants what accent they would like a robot to have. Participants responded with a wide range of accents, indicative of the fact that individual differences likely shape this preference - excluding gender and age, which did not influence it. Notably though, the biggest proportion of participants answered that they wanted a robot to have a Standard Southern British English accent (SSBE), followed by an Irish accent. This is in line with decades of Sociolinguistic studies in the UK, showing that British people find the SSBE accent trustworthy,

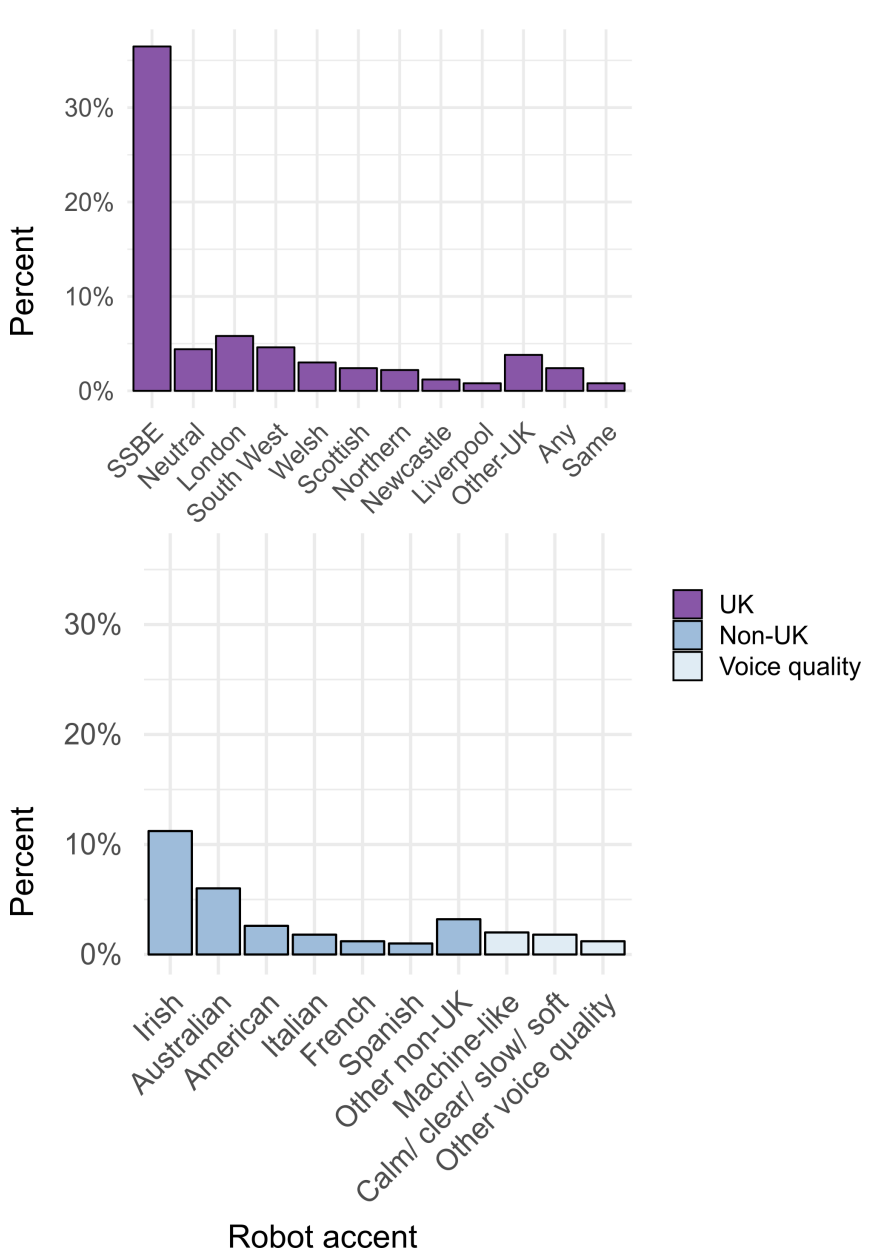

Fig. 2. Survey responses, standardised to fit wider categories of native British English accents (top), non-native English accents (bottom left) and different voice descriptions (bottom right)

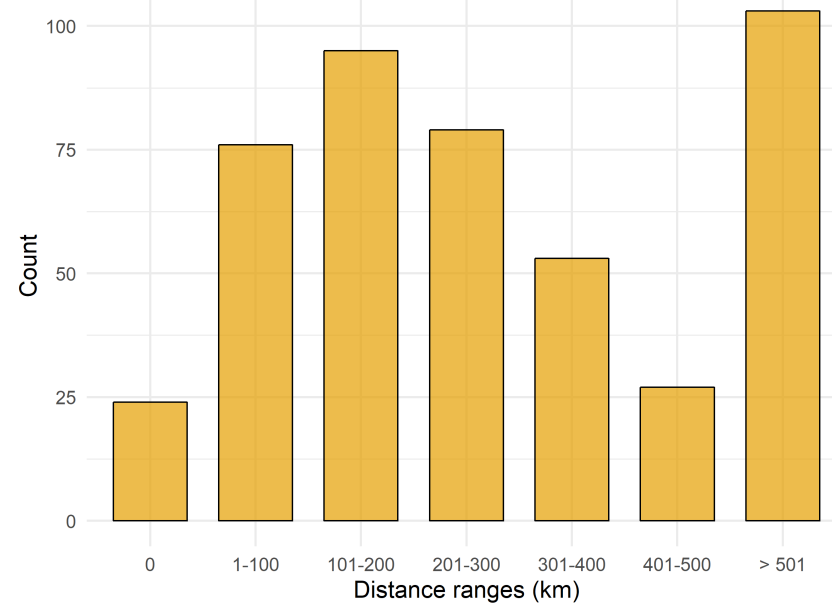

Fig. 3. Distances in km from participants' place of origin and participants' preferred location of a robot accent, divided in $100-\mathrm{km}$ intervals. The leftmost column (' 0 ') represents the number of people who indicated a robot accent corresponding to their same place of origin. 
socially attractive, and prestigious [e.g. 11]. The majority of participants might have felt that these characteristics were important in a robot. Linguists and the media have been working to de-stigmatise regional accents in the UK, for example with projects such as BBC Voices 1 , or by removing the requirement that all major news broadcasters speak with an SSBE accent 2 . However, as the current results demonstrate, accent attitudes still persist, especially when comparing regional and standard accents, and many people find it beneficial to 'not have an accent'. We had hypothesised that people's explicit accent preferences for a robot might be influenced by either in-group favouritism, or social constructions of prestige. Our results indicate that the latter might be at play. However, explicit preferences do not always correlate with experience-based impressions [30, 17] or with actual behaviour [27]. Thus, while it is important to keep user's preferences and expectations into account while designing a robot voice, future studies should also look at whether these explicit preferences are maintained in a real interaction scenario, and how they interact with other characteristics of the robot, such as behaviour or appearance.

A limitation of this survey is that the question was openended, so that the answers had to be re-coded by the authors to fit into standard categories. The biggest response category, 'SSBE', comprises several different terms that we assumed to refer to this particular accent (e.g. 'English', 'Southern', 'RP', 'British', 'general English', 'traditional English', 'no accent', 'well-spoken', 'proper English'). It is possible that the 'SSBE' label that we used diverges from what the participants intended. However, all the terms that were used share similar connotations on how the voice should sound, so we would still argue that all these terms are related, and that, for lack of a better term, they can be categorised as 'SSBE'. Another limitation is that, due to the open-ended nature of the demographics questionnaire, a few people indicated their region of origin, rather than city of origin; thus, the distance from home town calculation was not always accurate.

As for the second choice, Irish accent, this also traditionally receives positive ratings in Sociolinguistic surveys [e.g. 11], including winning the title of 'most attractive' accent of English in a government poll in 2014 [31].

These results seem to suggest that our participants had a clear idea of how they would like a robot to be accented. Most previous studies on accent evaluation are conceptual, i.e. they ask participants to rate a certain accent label in terms of a set of characteristics. Here we employed a sort of 'inverse conceptual' technique, because participants were not given any labels, but were asked to generate a label themselves. Thus, it is possible that they generated a label with geographic categorisations in mind, rather than with ideas of how a specific accent sounds. They might have thought about what connotations a certain accent, such as

\footnotetext{
${ }^{1} \mathrm{http} / / / \mathrm{www} . \mathrm{bbc} . \mathrm{co} . \mathrm{uk} / \mathrm{voices} /$, now archived
${ }^{2}$ see the call for a more diverse representation of British accents by the BBC chief in 2008: https://www.telegraph.co.uk/news/uknews/1575790/BBC-chief-calls-formore-regional-accents.html
}

SSBE or Irish, entails; and they might have assigned this label with these stereotypes in mind, because they wanted a robot to fulfil these characteristics. A meaningful next step in this line of research would be to make people interact with a robot speaking in some of the most and least preferred accents, and evaluate the interaction, to see if this categorisation holds when the geographic label is removed.

Only around $5 \%$ of participants indicated that they wanted the robot to have the same accent as their own (this figure drops to $0.8 \%$ if we consider the explicit answer, instead of the distance from home town). This does not support the hypothesis that people's preference for a robot accent might manifest itself in terms of in-group favouritism (Section [II). However, it seems that different traits evoke different explicit accent preferences [17, 6]. It is therefore possible that, when asked about a generic preference, participants thought of positive connotations afforded by having a standard accent, rather than specific positive same-group characteristics. It would be interesting to see people's answers to a question more focused on interpersonal relationships, such as: "What accent would you like your robot companion to have?".

The current results seem to contradict previous studies in Human-Agent Interaction, where people engaged more with agents that had their same accent [17, 22]. However, this apparent contradiction might simply be another reminder that collecting evaluations from humans is not trivial, and that people often end up behaving differently than what their explicit evaluations suggested [30]. Also, as previously mentioned, many people in the UK think that they do not have an accent. Therefore, the wording of our question might have primed them to think about other accents, i.e. not considering their own as one. Another possibility is that, if a layperson's 'no accent' equals what linguists call 'SSBE accent', it is also possible that our results actually indicate that the majority of participants really wanted the robot to have their same 'non-accent'. To the best of our knowledge, this is the first study explicitly asking participants what accent they would prefer on a robot, so it remains to be seen whether people will actually show a similar preference in real Human-Robot Interaction scenarios. For now, the explicit preferences we have collected do not not provide evidence of in-group favouritism.

Regarding the third hypothesis, only $2 \%$ of participants mentioned that they wanted a robot to have a machine-like voice. This goes against many practical recommendations within the field of HRI, which warn against the unrealistic expectations that human-like features on a robot, such as accent, might generate [7, 27, 29]. Again, we might be at an impasse between users' explicit preferences and practical considerations on interaction success. In fact, machine-like voices have already been shown to increase this success. For example, [2] conducted an experiment with human and machine-sounding phone service operators, and found that interactions with the machine-sounding voice were more efficient than those with the human voice. However, the interactions were not evaluated by the callers, so it is unclear whether they would have preferred to engage in a 
lengthier, but perhaps more human-like, phone call. Perhaps this impasse can be solved by giving robots synthetic accents. This might still help set the right expectations on the robot's capabilities, while at the same time giving the user a geographic frame of reference.

Despite the increasing quality of synthetic speech, dealing with regional accents is still a relatively unexplored topic. Generally, the approach consists in creating a corpus, or a set of rules encoded in a phonetic module, for each specific accent. Even if the literature is quite sparse, some resources and studies focused on a more flexible approach to render speech with a dedicated regional accent. The most well-known resource is the VoiceBank corpus [32], which contains speech samples from 500 speakers from several regions across Great Britain. However, so far this corpus has mostly been used for spoofing, speech in noise, and speech enhancement studies.

An interesting approach by [33] is to model a continuous accent space using joint sequence models. Each space is associated with a model, and from there the final goal is to be able to interpolate between the different models; hence the different accent spaces. However, this accent-based synthesis approach has not been validated with human listeners yet.

Even though recent innovations have been leading synthesis systems into producing human-like speech, the flexibility required to render natural human-like speech remains a difficult problem. Additionally, speech synthesis cannot be taken as a general problem with one solution fitting everyone [34]. This is the reason why synthesising expressive speech, as well as adapting the target speech to a given speaker, are the current hot challenges of the community. Consequently, rendering speech with a proper accent is an important step that will attract speech technologists rather sooner than later. These forthcoming advances in speech technology will hopefully increase the HRI community's interest in robot voices and will allow to create distinct regional language varieties while controlling for the desired level of machinelikeness.

Thus, giving robots synthetic accents could be a promising line of research to see whether we can satisfy both arguments: a noticeable machine quality in the voice, in order to set the right expectations about the robot capabilities; and a salient human geographic label, in order to create a frame of reference shared by both human and robot.

Finally, a limitation that must be mentioned is that participants in this study were all from the UK. While our results can be informative when dealing with robot voices for British users, they cannot be generalised to users outside of the UK. More surveys from other countries are needed to make this generalisation, and it would be especially interesting to see how speakers of languages with a clearly defined standard accent compare to speakers of languages without one.

\section{CONCLUSION}

In this paper, we presented subjective preferences of over 500 people from the UK towards a robot accent.The biggest proportion of respondents stated that they would like the robot to have a Standard Southern British English accent, followed by an Irish accent. The results also suggest that only a few people would prefer the robot to have a robot-like voice, or an accent like their own. While many of today's manufacturers of speaking robots give them a default voice, it is important to bear in mind that more variability in robot voices might resonate more with users' preferences. While we do not claim that robots should have an SSBE or Irish accent, we suggest that robot manufacturers should pay attention to the voice of their robots, as potential users also seem to do.

\section{REFERENCES}

[1] M. P. Aylett, S. J. Sutton, and Y. Vazquez-Alvarez, "The right kind of unnatural: Designing a robot voice," in Proceedings of the 1st International Conference on Conversational User Interfaces, ser. CUI '19, 2019, pp. $1-2$.

[2] R. K. Moore, "Appropriate voices for artefacts: some key insights," in 1st International Workshop on Vocal Interactivity in-and-between Humans, Animals and Robots, ser. VIHAR, 2017.

[3] M. West, R. Kraut, and H. Chew. (2019) I'd blush if i could: closing gender divides in digital skills through education. [Online]. Available: https: //unesdoc.unesco.org/ark:/48223/pf0000367416

[4] K. D. Kinzler, K. H. Corriveau, and P. L. Harris, "Children's selective trust in native-accented speakers," Developmental Science, vol. 14, no. 1, pp. 106-111, 2011.

[5] R. Lippi-Green, English with an accent: Language, ideology, and discrimination in the United States. Psychology Press, 1997.

[6] H. Giles, "Patterns of evaluation to RP, South Welsh and Somerset accented speech," British Journal of Social and Clinical Psychology, vol. 10, no. 3, pp. 280281, 1971.

[7] S. Wilson and R. K. Moore, "Robot, alien and cartoon voices: Implications for speech-enabled systems," in $1 s t$ International Workshop on Vocal Interactivity in-andbetween Humans, Animals and Robots, ser. VIHAR, 2017, pp. 40-44.

[8] D. S. Syrdal, K. Dautenhahn, K. L. Koay, M. L. Walters, and W. C. Ho, "Sharing spaces, sharing livesthe impact of robot mobility on user perception of a home companion robot," in International Conference on Social Robotics. Springer, 2013, pp. 321-330.

[9] S. H. Seo, D. Geiskkovitch, M. Nakane, C. King, and J. E. Young, "Poor thing! would you feel sorry for a simulated robot? a comparison of empathy toward a physical and a simulated robot," in Proceedings of the 10th Annual International Conference on Human-Robot Interaction, ser. HRI '15. ACM/IEEE, 2015, pp. 125132.

[10] H. Giles, "Evaluative reactions to accents," Educational review, vol. 22, no. 3, pp. 211-227, 1970.

[11] H. Bishop, N. Coupland, and P. Garrett, "Conceptual 
accent evaluation: Thirty years of accent prejudice in the UK," Acta Linguistica Hafniensia, vol. 37, no. 1, pp. 131-154, 2005.

[12] S. Lev-Ari and B. Keysar, "Why don't we believe nonnative speakers? the influence of accent on credibility," Journal of Experimental Social Psychology, vol. 46, no. 6, pp. 1093-1096, 2010-11.

[13] K. D. Kinzler, K. Shutts, J. M. DeJesus, and E. S. Spelke, "Accent trumps race in guiding children's social preferences," Social Cognition, vol. 27, no. 4, pp. 623634, 2009.

[14] T. Nazzi, J. Bertoncini, and J. Mehler, "Language discrimination by newborns: toward an understanding of the role of rhythm." Journal of Experimental Psychology: Human perception and performance, vol. 24, no. 3, p. 756, 1998.

[15] A. C. Cargile and H. Giles, "Understanding language attitudes: Exploring listener affect and identity," Language \& Communication, vol. 17, no. 3, pp. 195-217, 1997.

[16] K. D. Kinzler, K. Shutts, and J. Correll, "Priorities in social categories," European Journal of Social Psychology, vol. 40, no. 4, pp. 581-592, 2010.

[17] R. Tamagawa, C. I. Watson, I. H. Kuo, B. A. MacDonald, and E. Broadbent, "The effects of synthesized voice accents on user perceptions of robots," International Journal of Social Robotics, vol. 3, no. 3, pp. 253-262, 2011.

[18] H. Tajfel, M. G. Billig, R. P. Bundy, and C. Flament, "Social categorization and intergroup behaviour," European Journal of Social Psychology, vol. 1, no. 2, pp. 149-178, 1971.

[19] C. I. Nass, B. J. Fogg, and Y. Moon, "Can computers be teammates?" International Journal of HumanComputer Studies, vol. 45, no. 6, pp. 669-678, 1996.

[20] J. L. Marble, D. J. Bruemmer, D. A. Few, and D. D. Dudenhoeffer, "Evaluation of supervisory vs. peer-peer interaction with human-robot teams," in Proceedings of the 37th Annual Hawaii International Conference on System Sciences. IEEE, 2004.

[21] C. McGinn and I. Torre, "Can you tell the robot by the voice? an exploratory study on the role of voice in the perception of robots," in Proceedings of the 14th Annual International Conference on Human-Robot Interaction, ser. HRI '19. ACM/IEEE, 2019, pp. 211-221. [Online]. Available: https://doi.org/10.1109/ HRI.2019.8673305

[22] N. Dahlbäck, S. Swamy, C. Nass, F. Arvidsson, and J. Skågeby, "Spoken interaction with computers in a native or non-native language-same or different," in Proceedings of INTERACT, 2001, pp. 294-301.

[23] N. Spatola, N. Anier, S. Redersdorff, L. Ferrand, C. Belletier, A. Normand, and P. Huguet, "National stereotypes and robots' perception: The "made in" effect," Frontiers in Robotics and AI, vol. 6, p. 21, 2019.

[24] S.-1. Lee, I. Y.-m. Lau, S. Kiesler, and C.-Y. Chiu, "Human mental models of humanoid robots," in Pro- ceedings of the 2005 IEEE international Conference on Robotics and Automation, ser. ICRA '05. IEEE, 2005, pp. 2767-2772.

[25] S. Andrist, M. Ziadee, H. Boukaram, B. Mutlu, and M. Sakr, "Effects of culture on the credibility of robot speech," in Proceedings of the 10th Annual International Conference on Human-Robot Interaction, ser. HRI '15. ACM/IEEE, 2015, pp. 157-164.

[26] J. Cambre, J. Maddock, J. Tsai, J. Colnago, and J. Kaye, "Choice of voices: A large-scale evaluation of text-tospeech voice quality for long-form content," in Proceedings of the 2020 SIGCHI Conference on Human Factors in Computing Systems, ser. CHI '20, 2020.

[27] I. Torre, J. Goslin, L. White, and D. Zanatto, "Trust in artificial voices: A "congruency effect" of first impressions and behavioural experience," in Proceedings of APAScience '18: Technology, Mind, and Society (TechMindSociety '18), 2018.

[28] M. Strait, L. Vujovic, V. Floerke, M. Scheutz, and H. Urry, "Too much humanness for human-robot interaction: exposure to highly humanlike robots elicits aversive responding in observers," in Proceedings of the 33rd annual ACM conference on human factors in computing systems, 2015, pp. 3593-3602.

[29] W. J. Mitchell, K. A. Szerszen, A. S. Lu, P. W. Schermerhorn, M. Scheutz, and K. F. MacDorman, "A mismatch in the human realism of face and voice produces an uncanny valley," $i$-Perception, vol. 2 , no. 1 , pp. 10-12, 2011.

[30] A. G. Greenwald and M. R. Banaji, "Implicit social cognition: attitudes, self-esteem, and stereotypes." Psychological Review, vol. 102, no. 1, p. 4, 1995.

[31] YouGov.co.uk. (2014, feb) Survey results. [Online]. Available: http://cdn.yougov. com/cumulus_uploads/document/iwkrmxbxyl/ InternalResults_141128_accents_Website.pdf

[32] C. Veaux, J. Yamagishi, and S. King, "The voice bank corpus: Design, collection and data analysis of a large regional accent speech database," in 2013 International Conference Oriental COCOSDA held jointly with 2013 Conference on Asian Spoken Language Research and Evaluation (O-COCOSDA/CASLRE). IEEE, 2013, pp. $1-4$.

[33] B. Kolluru, V. Wan, J. Latorre, K. Yanagisawa, and M. J. Gales, "Generating multiple-accent pronunciations for TTS using joint sequence model interpolation," in Fifteenth Annual Conference of the International Speech Communication Association, 2014, pp. 12731277.

[34] P. Wagner, J. Beskow, S. Betz, J. Edlund, J. Gustafson, G. E. Henter, S. L. Maguer, Z. Malisz, É. Székely, C. Tånnander, et al., "Speech Synthesis Evaluation-State-of-the-Art Assessment and Suggestion for a Novel Research Program," in Speech Synthesis Workshop (SSW), 2019. 